\title{
A Case Report of Sandhoff Disease
}

\author{
R. Saouab • M. Mahi $\cdot$ R. Abilkacem • H. Boumdin • \\ S. Chaouir $\cdot$ O. Agader $\cdot$ T. Amil $\cdot$ A. Hanine
}

Received: 30 May 2010 / Accepted: 9 September 2010 / Published online: 10 December 2010

(C) The Author(s) 2010. This article is published with open access at Springerlink.com

\begin{abstract}
Sandhoff disease is a rare and severe lysosomal storage disorder representing 7\% of GM2 gangliosidoses. Bilateral thalamic involvement has been suggested as a diagnostic marker of Sandhoff disease. A case of an 18-month-old infant admitted for psychomotor regression and drug resistant myoclonic epilepsy is presented. Cerebral CT scan showed bilateral and symmetrical thalamic hyperdensity. MRI revealed that the thalamus was hyperintense on $\mathrm{T}_{1}$-weighted images and hypointense on T2-weighted images with a hypersignal T2 of the white matter. Enzymatic assays objectified a deficiency of both hexosaminidases $\mathrm{A}$ and $\mathrm{B}$ confirming the diagnosis of Sandhoff disease.
\end{abstract}

Keywords Computed tomography $\cdot$ Magnetic resonance imaging $\cdot$ Sandhoff disease $\cdot$ Thalamic hyperdensity

\section{Introduction}

Sandhoff disease is a rare but severe lysosomal storage disorder caused by a deficiency of both hexosaminidases A and $\mathrm{B}$ resulting in accumulation of glycosphingolipids and oligosaccharides in the brain. It represents $7 \%$ of cases of

R. Saouab $(\bowtie) \cdot$ M. Mahi $\cdot$ H. Boumdin $\cdot$ S. Chaouir

T. Amil $\cdot$ A. Hanine

Department of Radiology, Mohamed 5th Military Hospital,

Rabat, Morocco

e-mail: rachida_sa@yahoo.fr

R. Saouab $(\bowtie)$

CYM, Hay el Fath, IM 198 - App 8, Rabat, Morocco

R. Abilkacem · O. Agader

Department of Pediatrics, Mohamed 5th Military Hospital,

Rabat, Morocco
GM2 gangliosidosis. Bilateral thalamic involvement has been suggested as a diagnostic marker of Sandhoff disease but other structures such as basal ganglia, white matter and cerebellum can also be involved. In this article a new case of this disease with typical thalamic involvement is reported.

\section{Observation}

An 18-month-old boy born after an uncomplicated full-term pregnancy was hospitalized for psychomotor regression and drug-resistant myoclonic epilepsy. There was a consanguinity of the first degree of the parents and the death of a brother at the age of 16 months under unspecified circumstances. The other brother and sister were unaffected.

The onset of clinical symptoms began at the age of 6 months with myoclonus of the face and upper limbs, recurrent fever and psychomotor developmental delay. Physical examination revealed a macrocephaly of $51 \mathrm{~cm}$ with a dysmorphic syndrome consisting of a frontal bossing and a broadening of the nasal bridge. The neurological examination revealed an axial hypotonia with no further optical problems or responsiveness to light. Ophthalmologic examination showed a cherry-red spot without optic atrophy.

Cerebral computed tomography (CT) scanning performed without contrast medium injection showed a bilateral thalamic hyperdensity with hypodensity of the white matter (Fig. 1).

With magnetic resonance imaging (MRI) the thalamus was hyperintense on T1-weighted images and hypointense on T2-weighted images (Fig. 2) with an increased T2 signal of the white matter.

No imaging changes could be observed in the caudate nucleus, putamen, globus pallidum and brainstem. 
Fig. 1 Cerebral CT scan without contrast medium injection showing a bilateral thalamic hyperdensity with hypodensity of the white matter
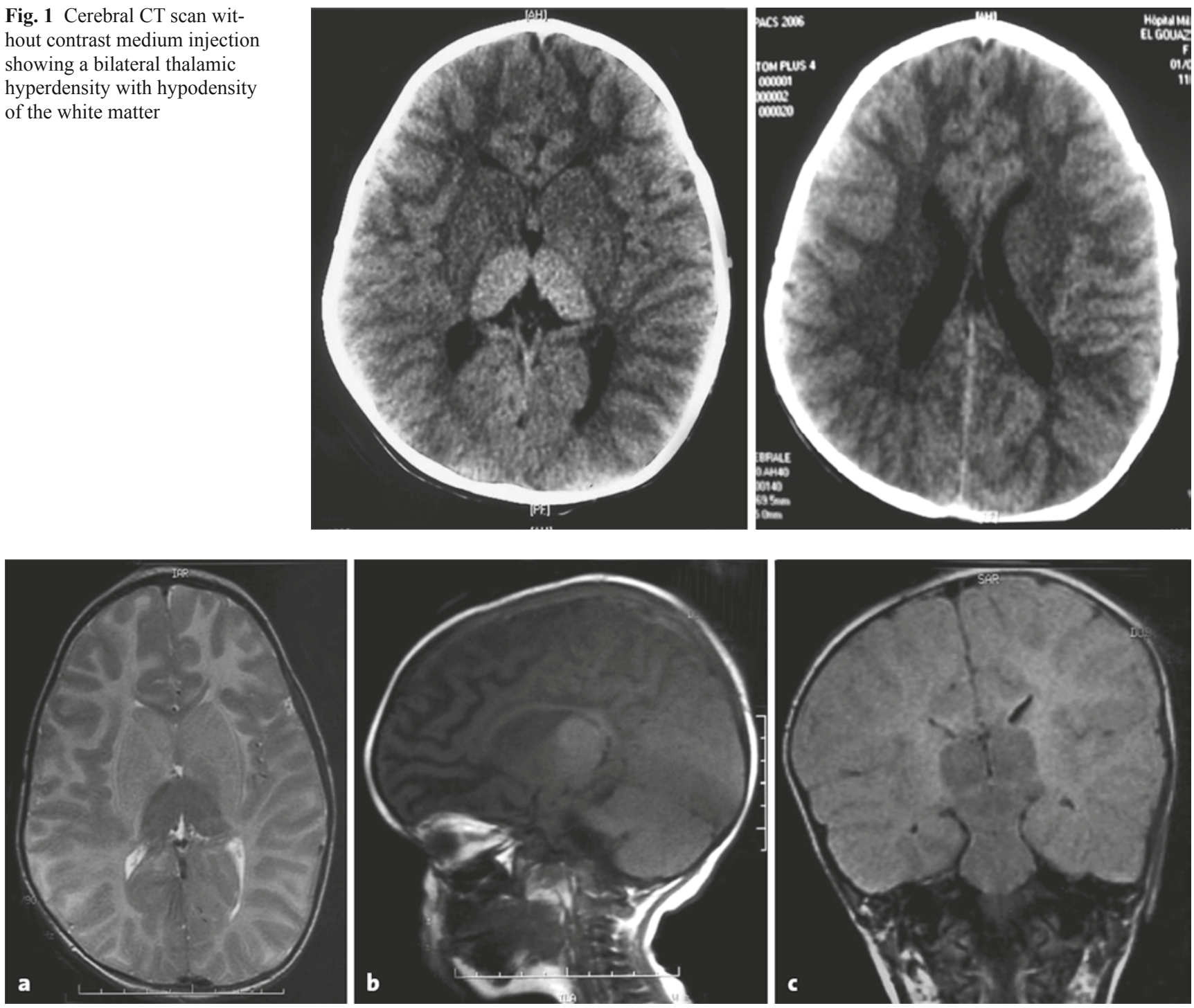

Fig. 2 Axial T2 (a) sagittal T1 (b) and coronal FLAIR T2-weighted MR images (c) demonstrating T2 hypointensity and T1 hyperintensity of the thalamus with $\mathrm{T} 2$ hyperintensity of the white matter

Clinical data and thalamic involvement suggest metabolic diseases such GM2 gangliosidosis (e.g. Tay-Sachs and Sandhoff diseases) or GM1 gangliosidosis (e.g. Landing disease), Canavan disease or Alexander disease.

Chromatography of amino acids in the blood, urine and cerebrospinal fluid (CSF) was normal and chromatography of organic acids in the urine was also normal. Enzymatic assays were performed in France and revealed a deficiency of both hexosaminidases A and B confirming the diagnosis of Sandhoff disease.

\section{Discussion}

Sandhoff disease is a rare autosomal recessive inherited metabolic disorder caused by mutations in the HEXB gene on chromosome 5q13. It was first described by Konrad Sandhoff in 1968.

There are three different forms of Sandhoff disease: classic infantile, juvenile and adult late onset. Classic infantile form is the most common and severe form, clinical onset is generally between 3 and 9 months of age after a normal development and is characterized by muscular hypotonia and tonico-clonic or myoclonic seizures, blindness, psychomotor retardation and paralysis [1]. A cherry-red spot in macular areas is characteristic but not specific. Juvenile and adult onset forms of Sandhoff disease are very rare. Signs and symptoms can begin in childhood, adolescence or adulthood and are usually milder than those seen with the infantile form of Sandhoff disease. No treatment is currently available and patients with infantile types usually die before 3 years of age [2]. 
Cerebral images of GM2 gangliosidosis have been published in a few reports and bilateral homogeneous thalamic hyperdensity on CT was reported to be an early sign [3]. Loss of axons and myelin in the central cortical neurons, gliosis and intralysosomal storage in addition to calcium accumulation might be responsible for this appearance [4].

Magnetic resonance imaging (MRI) typically reveals hypo-intensity in T2-weighted images of the thalamus [5] and high signal abnormalities have been reported in T2weighted sequences in the caudate nucleus, globus pallidus and putamen [6]. Also the cerebral white matter shows homogenous or patchy high signal intensity which suggests a combination of disturbed and abnormal myelination and myelin loss. An unusual presentation of Sandhoff disease was reported by Nassogne et al. with diffuse signal changes exclusively in the brainstem of a 3-year-old girl [7]. Brainstem involvement appeared as a construction from the internal capsule involvement and descended downward along the long fiber tracts of the pons through the crus cerebri.

Recently, Lowe et al. found an additional $2.07 \mathrm{ppm}$ resonance in proton MRI spectra of the brain of Sandhoff mice which could be identified as originating from $\mathrm{N}$-acetylhexosamine [8]. Wilken et al. reported similar proton MRS findings in a child with enzymatically proven Sandhoff disease. There was a reduction of total $\mathrm{N}$-acetylaspartate (neuroaxonal marker) as well as strongly elevated inositol levels (glial marker) in white matter, gray matter and basal ganglia [9].

A decrease in T2 signal intensity in the thalami seems to be a sign of lysosomal diseases [10] and may also be identified in Tay-Sachs disease GM1 gangliosidoses and in late stages of Canavan disease or Krabbe's disease. Patients with late stage Canavan disease may have dense thalami on T1weighted images, as well as white matter necrosis, resulting in cavitation and brain stem and cerebellar atrophy, which are not observed in GM1 gangliosidoses. In Krabbe's disease many more brain structures may demonstrate similar high density on $\mathrm{CT}$ and white matter disease is more extensive, more homogenous and does not spare the corpus callosum [4].

Diagnosis is confirmed by enzymatic assays revealing a deficiency of both lysosomal hydrolase $\beta$ hexosaminidases A and B. In summary Sandhoff disease is a rare inherited lysosomal storage disorder characterized by thalamic hyperdensity which may be a useful sign for the differential diagnosis of neurodegenerative disorders with a cherry-red spot at the macula. Currently, proton magnetic resonance spectography (MRS) of cerebral metabolites may prove to represent another disease-specific MRS pattern of the brain.

Open Access This article is distributed under the terms of the Creative Commons Attribution Noncommercial License which permits any noncommercial use, distribution, and reproduction in any medium, provided the original author(s) and the source are credited.

\section{References}

1. Hendriksz CJ, Corry PC, Wraith JE, Besley GTN, Cooper A, Ferrie CD. Juvenile Sandhoff disease - nine new cases and a review of the literature. J Inherit Metab Dis. 2004;27:241-9.

2. Yüksel A, Yalçmkaya C, Islak C, Gündüz E, Seven M. Neuroimaging findings of four patients with Sandhoff disease. Pediatr Neurol. 1999;21:562-5.

3. Gutiérrez-Solana LG, Ruiz-Falcó ML, García-Peñas JJ, Chabás A, Manzano-Blanco S, Gómez-Mardones G, López-Terradas JM. Evolutive neuroradiological alterations in Sandhoff's disease. Rev Neurol. 1996 Oct;24(134):1263-6.

4. GM2 gangliosidosis. In: van der Knaap MS, Valk J, editors. Magnetic resonance of myelin, myelination, and myelin disorders. Springer, Berlin; 1995. pp. 81-9.

5. Kölfen W, Freund M, Jaschke W, König S, Schultze C. GM-2 gangliosidosis (Sandhoff's disease): two year follow-up by MRI. Neuroradiology. 1994;36:152-4.

6. Hittmair K, Wimberger D, Bernert G, Mallek R, Schindler EG. MRI in a case of Sandhoff's disease. Neuroradiology. 1996;38 Suppl 1:178-80.

7. Nassogne M-C, Commare M-C, Lellouch-Tubiana A, Emond S, Zerah M, Caillaud C, Hertz-Pannier L, Saudubray J-M. Unusual presentation of GM2 gangliosidosis mimicking a brain stem tumor in a 3-year-old girl. AJNR Am J Neuroradiol. 2003;24:840-2.

8. Lowe JP, Stuckey DJ, Awan FR, Jeyakumar M, Neville DCA, Platt FM, Griffin JL, Styles P, Blamire AM, Sibson NR. MRS reveals additional hexose $\mathrm{N}$-acetyl resonances in the brain of a mouse model for Sandhoff disease. NMR Biomed. 2005;18:517-26.

9. Wilken B, Dechent P, Hanefeld F, Frahm J. Proton MRS of a child with Sandhoff disease reveals elevated brain hexosamine. Eur J Paediatr Neurol. 2008;12:56-60.

10. Autti T, Joensuu R, Aberg L. Decreased T2 signal in the thalami may be a sign of lysosomal storage disease. Neuroradiology. 2007 Jul;49(7):571-8. 